AUTOR:

SérGio Podgaec

ORIENTADOR:

Prof. Dr. MAURICIO SimÕes ABRÃo

\title{
Padrões de resposta imune em pacientes com endometriose
}

\author{
Immune response patterns in patients with endometriosis
}

Resumo de tese

Palavras-chave

Endometriose Imunologia

Cirtocinas
Tese apresentada ao Programa de Pós-Graduação da Faculdade de Medicina da Universidade de São Paulo, Área de Concentração Obstetrícia e Ginecologia, para obtenção do Título de Doutor em Medicina, em 12 de setembro de 2006.

OBJETIVO: analisar a relação e a predominância das respostas imunes Th 1 e Th2 em pacientes com endometriose. MÉTODOS: entre fevereiro de 2004 e abril de 2005, foram avaliadas 98 pacientes divididas em dois grupos de acordo com a presença (Grupo A) ou ausência de endometriose (Grupo B), confirmada histologicamente. Foram coletados sangue periférico e fluido peritoneal de todas as pacientes para a dosagem de interleucinas (IL) 2, 4 e 10, fator de necrose tumoral-alfa (TNF-alfa) e interferon-gama (IFN-gama) por citometria de fluxo. Além da presença da endometriose, foram analisados a fase do ciclo menstrual, o quadro clínico, o estadiamento, o local de acometimento e a classificação histológica da moléstia. RESULTADOS: observou-se elevação significante nos valores de interferongama (mediana de 1,5 pg/mL no Grupo A e de 0,4 pg/mL no Grupo B, p=0,03) e de interleucina-10 (mediana de 38,6 pg/mL no Grupo A e de 15,7 pg/mL no Grupo B, p=0,03) medidos no fluido peritoneal das pacientes com endometriose em relação àquelas sem a doença. As pacientes com endometriose apresentaram alteração significativa na relação das concentrações de IL-4/IFN-gama (p<0,00 1), IL-4/IL-2 (p=0,006), IL-10/INF-gama (p<0,00 1) e IL-10/IL-2 $(p<0,001)$ do fluido peritoneal, com concentrações mais elevadas da IL-4 e da IL-10, o que reflete o predomínio da resposta Th2 sobre a Th 1. CONCLUSÃO: os resultados obtidos neste estudo permitem concluir que a endometriose é uma doença que apresenta características inflamatórias associadas a um padrão de resposta imune do tipo Th2.
AUTOR:

Lella Maria Geromel Dotto

ORIENTADOR:

Profa. Dra. Marli Villela Mamede

\section{Atenção qualificada ao parto: a realidade da assistência de enfermagem em Rio Branco (AC)}

\author{
Qiualified delivery care: the reality of nursing assistance in Rio Branco
}

Palavras-chave

Equipe de enfermagem

Parto

Enfermagem obstétrica Competência profissional.
Tese de Doutorado em Enfermagem apresentada a Escola de Enfermagem de Ribeirão Preto da Universidade de São Paulo, Departamento de Enfermagem Materno-Infantil e Saúde Pública, Programa de Pós-Graduação em Enfermagem em Saúde Pública, em 22 de setembro de 2006.

OBJETIVOS: identificar e descrever o perfil dos profissionais de enfermagem que atuam na atenção ao trabalho de parto e parto normal nas duas maternidades do município de Rio Branco (AC), e analisar as competências essenciais desenvolvidas por eles nesta prática. METODOLOGIA: estudo descritivo, com abordagem quantitativa. A população estudada foi composta por 30 profissionais de enfermagem. A coleta de dados foi realizada por meio de entrevistas e observações sistemáticas das competências essenciais em obstetrícia. Foram observados 14 admissões, 34 evoluções de trabalho de parto, 11 partos normais e 11 pós-parto. A estatística descritiva e o teste exato de Fisher foram usados para análise dos dados. RESULTADOS: perfil dos profissionais: a carga horária média semanal de trabalho foi de 63,3 horas, 40\% deles trabalham em mais de uma instituição. Dos 25 (83,3\%) profissionais que realizam parto normal, $18(72 \%)$ receberam treinamento informal para realizar o parto, os demais (7 ou 28\%) são enfermeiras obstétricas que foram capacitadas por meio de programas de pós-graduação lato sensu. Observou-se que com relação às competências essenciais, muitas das habilidades essenciais em obstetrícia deixaram de ser desenvolvidas ou, quando realizadas, aconteceram de forma incompleta. $\bigcirc$ modelo de divisão de trabalho para o desempenho das competências obstétricas de maior complexidade, nas instituições estudadas, mostrou diferenças significantes. CONCLUSÕES: de acordo com os critérios e requisitos estabelecidos pelas políticas internacionais sobre o atendimento qualificado ao parto, a realidade revela carência de pessoal qualificado. $\bigcirc$ modelo de atenção é caracterizado por uma divisão de trabalho que não privilegia a qualificação profissional. 